\title{
Author Correction: Moth biomass has fluctuated over 50 years in Britain but lacks a clear trend
}

\author{
Callum J. Macgregor (iD, Jonathan H. Williams, James R. Bell and Chris D. Thomas (D)
}

Correction to: Nature Ecology \& Evolution https://doi.org/10.1038/s41559-019-1028-6, published online 11 November 2019.

In the original version of this Brief Communication, software errors during data extraction and initial processing led to a substantial corruption of the version of the Rothamsted Insect Survey (RIS) dataset that was used in analyses. This corruption systematically affected a quarter of the total time series (1967-1979 and 2017, but not 1980-2016), and was responsible for the large increase in estimated biomass that was observed between 1978 and 1980 .

In light of these errors, all analyses presented in the original paper were rerun using the correct RIS data. The spirit of the two main conclusions in the abstract was largely unchanged. The primary conclusion, that 'severe declines [in insect biomass] are not supported by the world's longest-running insect population database', is still supported, although the original finding that moth biomass increased over the study period has been revised from 'moth biomass estimates ... revealed increasing biomass between 1967 and 1982 , followed by gradual decline from 1982 to 2017, with a 2.2-fold net gain in mean biomass between the first (1967-1976) and last decades (2008-2017) of monitoring.' to 'moth biomass estimates... revealed substantial between-year biomass change but no difference in mean biomass between the first (1967-1976) and last decades (2008-2017) of monitoring'. Analyses underpinning the second conclusion, that 'High between-year variability and multi-year periodicity in biomass emphasize the need for long-term data to detect trends and identify their causes robustly', were unaffected by the data corruption and therefore this conclusion is also still supported.

However, some of the fine detail of the results has changed. Analysing patterns in mean annual biomass per trap over time, the authors now find no significant differences between the first, last and peak decades of the time series (rather than a significant increase in biomass between the first and peak, and between the first and last decades, respectively). The linear trend of biomass between 1967 and
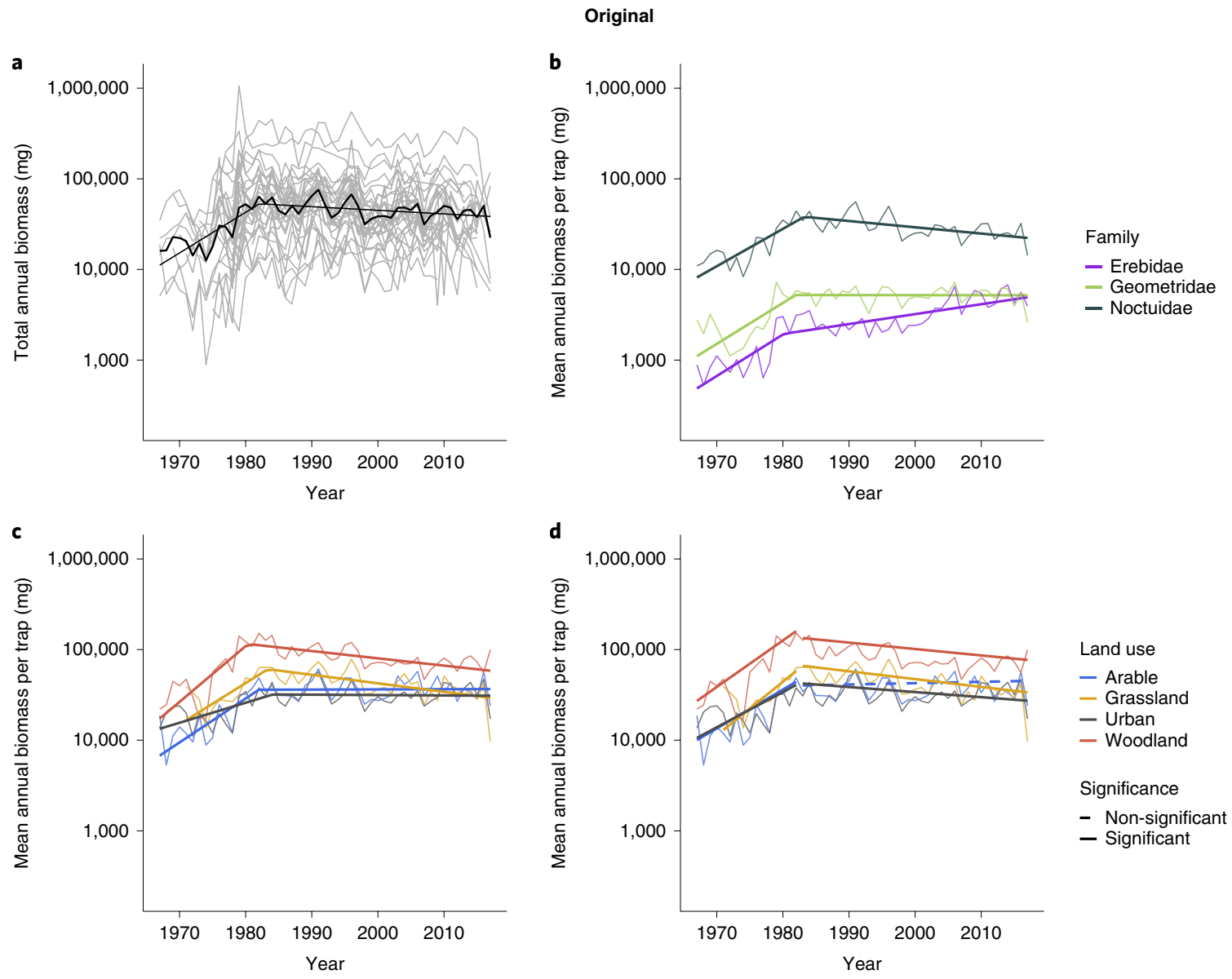

Fig. 1 | Original and Corrected. 
Corrected

a
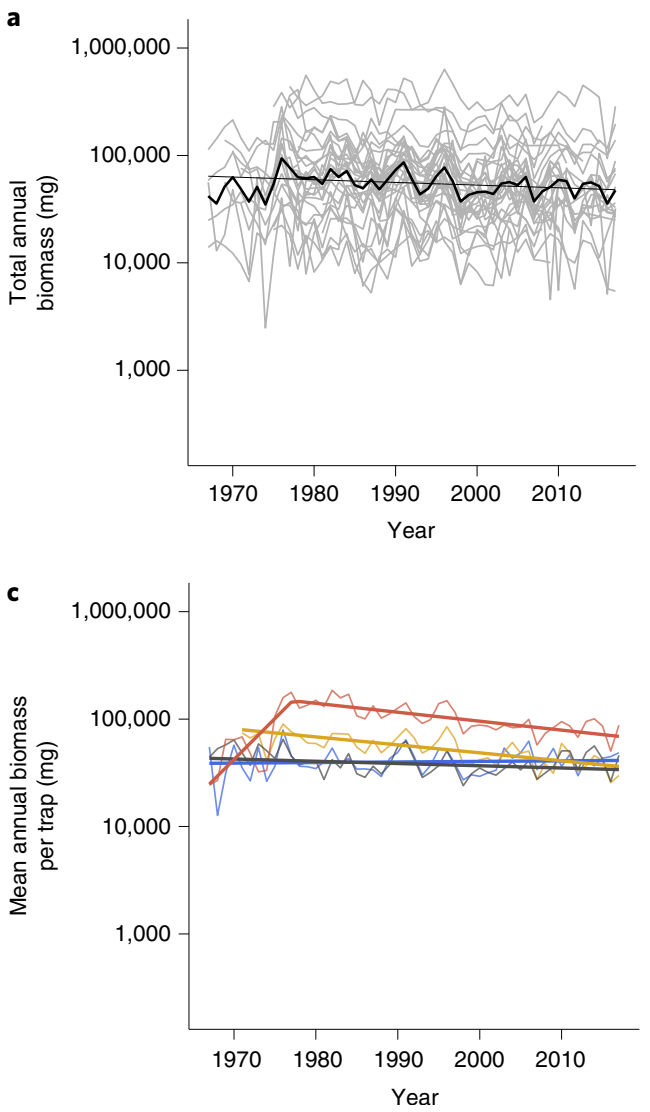

b

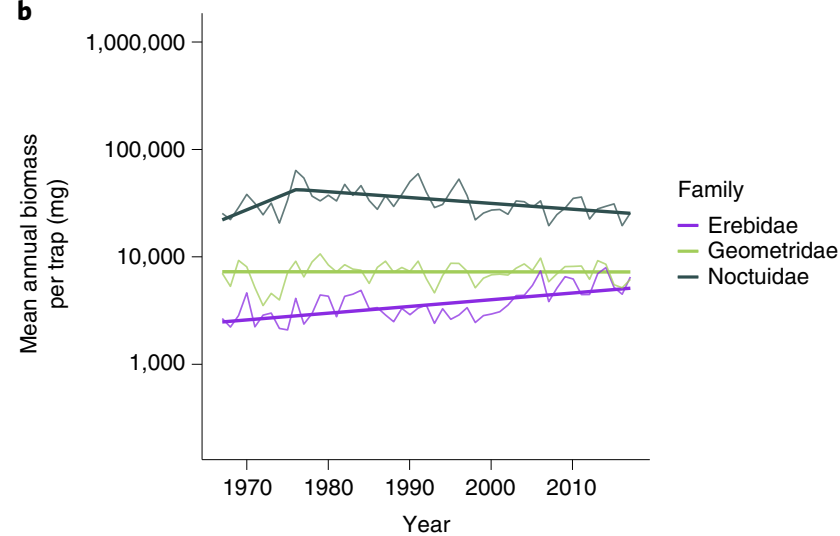

d

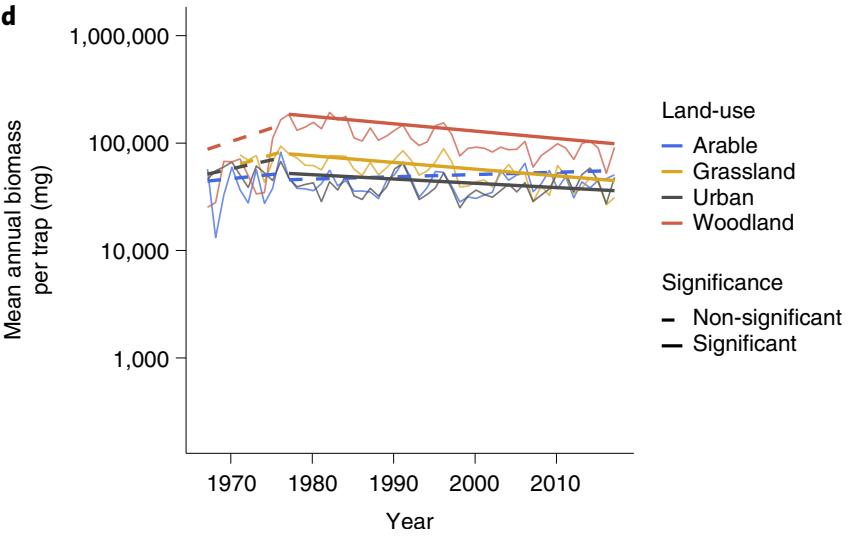

2017 across all traps is now significantly negative (rather than positive), and segmented regression now performs similarly to linear regressions (rather than outperforming them), with the peak in biomass now estimated to be around 1976 (rather than 1982). Analysing patterns independently for each trap site, four out of eight traps which commenced in 1970 or earlier were best-described by a segmented model that increased initially, then declined. We therefore now conclude that the post-1976 decline in the biomass of British moths was preceded by a comparable increase (rather than a larger increase).

Analysing patterns separately for different land-use types and moth families revealed less consistency than previously. However, the general pattern of increase followed by decline was still supported for Noctuidae (the family contributing the largest amount to assemblage biomass), the largest pre-1976 increases and post-1976 declines still took place in woodland and grassland land uses, and the authors still detected no post-1976 declines in biomass on arable land.

Specifically, the following text and figures were amended in the main article:

Third paragraph of main text, first sentence: 'Contrary to previous reports of insect biomass change, moth biomass increased before it declined and remains higher than in the late 1960s (Fig. 1).' was corrected to 'Contrary to previous reports of insect biomass change, moth biomass increased before it declined and remains at a comparable level to the late 1960s (Fig. 1).'

Third paragraph of main text, second sentence: 'Mean ( \pm s.e.m.) annual biomass per trap was 40,782.8 \pm 2,665.6 mg for 2008-2017, a $\mathbf{2 . 2}$-fold increase from $\mathbf{1 8 , 6 5 3 . 4} \pm \mathbf{1 , 6 3 9 . 9} \mathbf{m g}$ in $1967-1976$ (Welch's $t$-test, $n=20 ; \boldsymbol{t}=\mathbf{- 7 . 0 3}$, d.f. $=\mathbf{1 7 . 9 0 ,} \boldsymbol{P}<\mathbf{0 . 0 0 1}$ ) and the linear trend of biomass between 1967 and 2017 across all traps was significantly positive (Supplementary Table 1).' was corrected to 'Mean ( \pm s.e.m.) annual biomass per trap was $49,315.5 \pm 2,433.3 \mathrm{mg}$ for $2008-2017$, not significantly different from 48,915.9 $\pm \mathbf{5 , 5 4 5 . 5} \mathrm{mg}$ in 1967-1976 (Welch's $t$-test, $n=20 ; \boldsymbol{t}=\mathbf{- 0 . 0 7}$, d.f. $=\mathbf{1 3 . 9 3 ,} \boldsymbol{P}=\mathbf{0 . 9 4 1}$ ), but the linear trend of biomass between 1967 and 2017 across all traps was significantly negative (Supplementary Table 1 ).'

Third paragraph of main text, third sentence: 'However, segmented regression models (with a change in slope) outperformed linear regressions, indicating biomass peaked around 1982; mean ( \pm s.e.m.) annual biomass per trap for 1978-1987 was 46,790.1 \pm 3,670.4 $\mathrm{mg}$, significantly higher than in the first decade (Welch's $t$-test, $n=20 ; t=-7.49$, d.f. $=17.75, P<0.001$ ) but not the most recent (Welch's $\boldsymbol{t}$-test, $\boldsymbol{n}=\mathbf{2 0} \boldsymbol{t}=\mathbf{- 1 . 1 6}$, d.f. $=\mathbf{1 7 . 3 6}, \boldsymbol{P}=\mathbf{0 . 2 6 4}$ ).' was corrected to 'However, segmented regression models (with a change in slope) performed similarly to linear regressions, indicating biomass peaked around 1976; mean ( \pm s.e.m.) annual biomass per trap for 1972-1981 was $56,670.7 \pm 5,524.5 \mathrm{mg}$, but this was not significantly higher than either the first decade (Welch's $t$-test, $n=20$; $t=$ -1.09 , d.f. $=18.0, P=0.287$ ) or the most recent (Welch's $t$-test, $n=20 ; t=-1.29$, d.f. $=13.99, P=0.219$ ).'

Third paragraph of main text, fourth sentence: 'Slope values for mixed-effect models in the periods 1967-1982 and 1983-2017 were extremely similar to those from segmented regressions (Supplementary Table 1), indicating that our conclusions are robust to the 


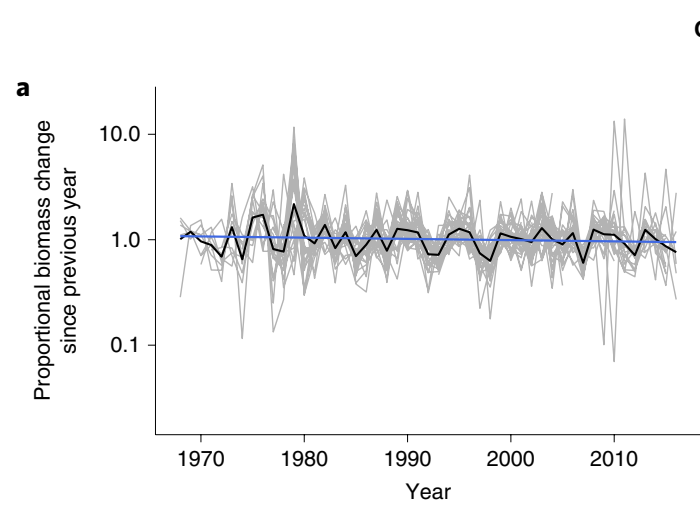

Original
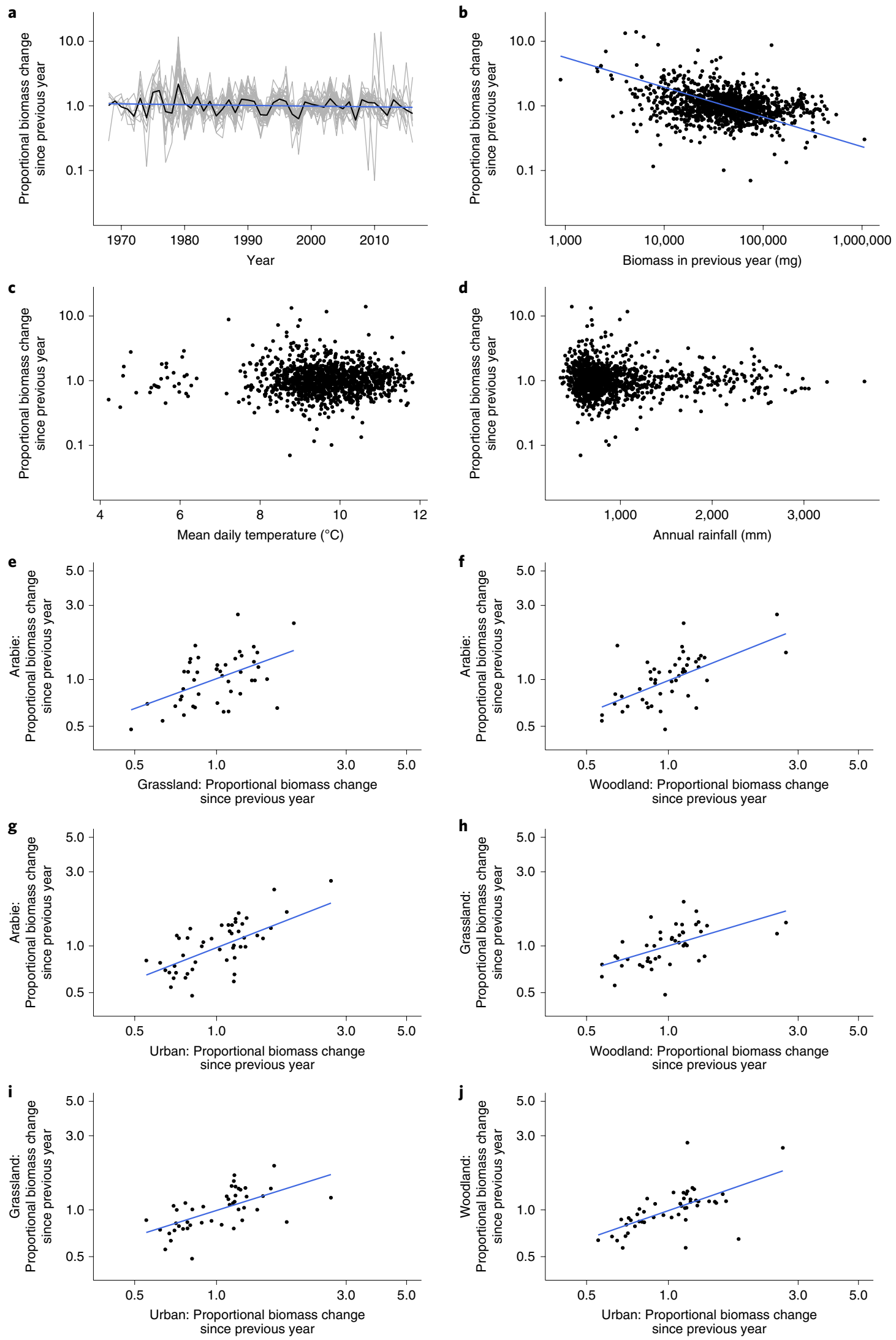

Fig. 2 | Original and Corrected. 
Corrected
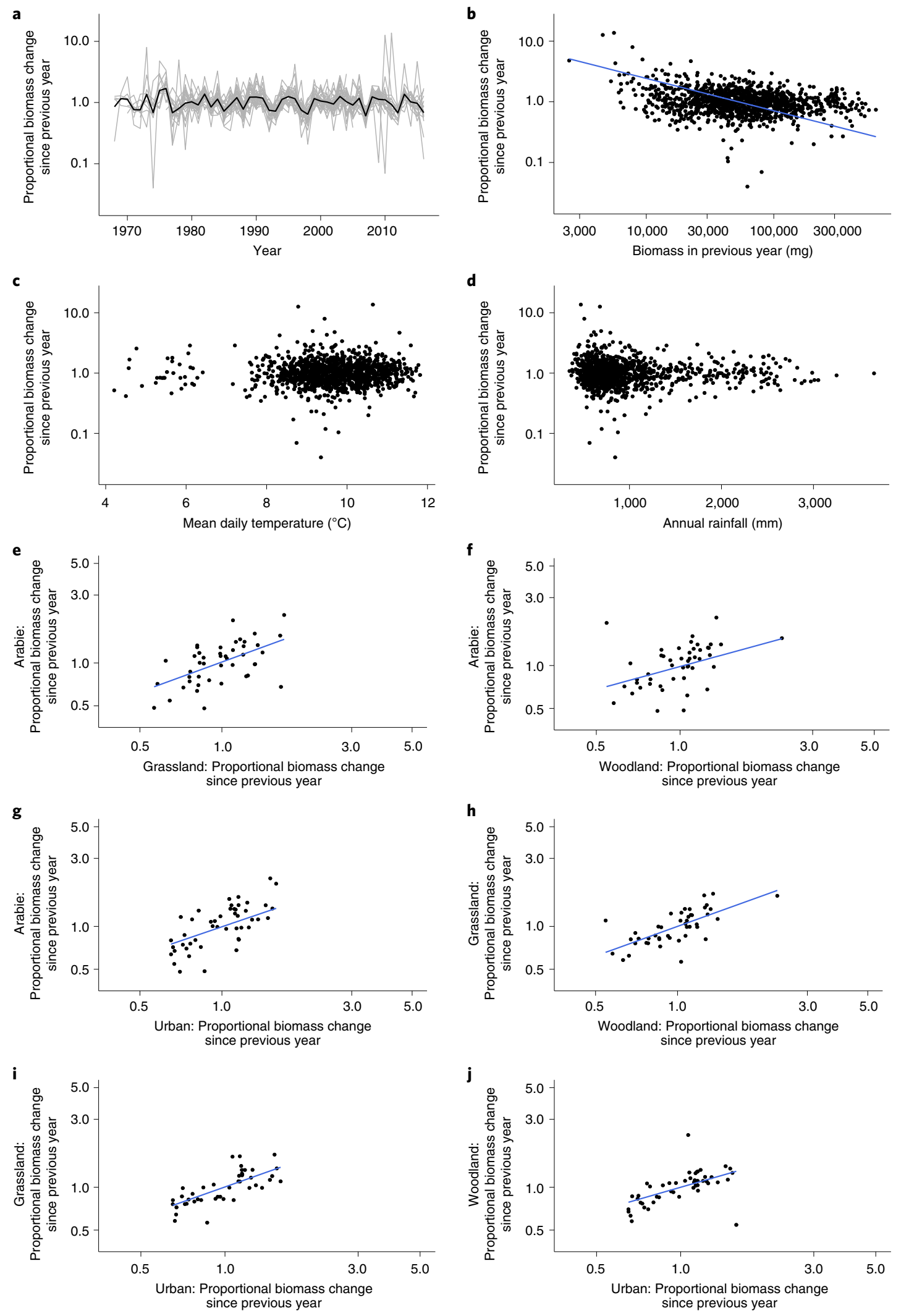

addition and removal of specific traps over time.' was corrected to 'Slope values for mixed-effect models in the periods 1967-1976 and 1977-2017 were generally similar to those from segmented regressions (Supplementary Table 1), indicating that our conclusions are robust to the addition and removal of specific traps over time.' 
Fourth paragraph of main text, second sentence: 'The general pattern of increase, followed by post-1982 decline was consistent across land uses for Noctuidae but other families showed different patterns in different land uses (Extended Data Fig. 2).' was corrected to 'The general pattern of increase, followed by post-1976 decline was supported for Noctuidae but other families showed different patterns in different land uses (Extended Data Fig. 2).'

Fourth paragraph of main text, third sentence: 'When biomass patterns were assessed independently for each trap, six out of eight traps (75\%) that ran for $>12$ yr before 1982 were best described by...' was corrected to 'When biomass patterns were assessed independently for each trap, four out of eight traps (50\%) which ran for $>6$ yr before 1976 were best described by...'

Fourth paragraph of main text, fourth sentence: 'The inflection took place at approximately the same time (Extended Data Fig. 4) regardless of the exact pattern of biomass change (for example, increase to $\sim 1982$ and stable thereafter, or post-1982 decline).' was corrected to 'The inflection took place at approximately the same time (Extended Data Fig. 4) for all models where segmented regression fitted as well as or better than linear regression, regardless of the exact pattern of biomass change (for example, increase to $\sim 1976$ and stable thereafter, or post-1976 decline).'

Fifth paragraph of main text, second sentence: 'Therefore, we separately considered biomass trajectories for traps that operated in woodland (the least intensively managed land use), grassland, arable land (which receives the highest chemical inputs) and urban areas (where light pollution is greatest), splitting the data into 1967-1982 and 1983-2017 periods, given the inflection point for the full model (Extended Data Fig. 4).' was corrected to 'Therefore, we separately considered biomass trajectories for traps that operated in woodland (the least intensively managed land use), grassland, arable land (which receives the highest chemical inputs) and urban areas (where light pollution is greatest), splitting the data into 1967-1976 and 1977-2017 periods, given the inflection point for the full model (Extended Data Fig. 4).'

Fifth paragraph of main text, third sentence: 'This reveals that the greatest pre-1982 increase took place in woodland and on arable land, followed by grassland, while the greatest post-1982 declines also took place in woodland and grassland (Fig. 1 and Supplementary Table 1), with no decline on arable land (Supplementary Table 2)'. was corrected to 'This reveals that the greatest pre-1976 increase took place in woodland, followed by grassland, while the greatest post-1976 declines also took place in woodland and grassland (Fig. 1 and Supplementary Table 1), with no decline on arable land (Supplementary Table 2).'

Fifth paragraph of main text, seventh sentence: 'However, our continuous sampling data at fixed sites revealed lower rates of decline (3.45\% increase to $\mathbf{1 8 . 0 0 \%}$ decline per decade, depending on land use; Supplementary Table 2) than were found by Hallmann et al. ${ }^{\text {.' }}$ was corrected to 'However, our continuous sampling data at fixed sites revealed lower rates of decline $(\mathbf{5 . 0 1 \%}$ increase to $14.59 \%$ decline per decade, depending on land use; Supplementary Table 2) than were found by Hallmann et al.'.'

Fifth paragraph of main text, eighth sentence: 'These post-1982 declines are lower than the 145.14-290.00\% per decade increases observed in 1967-1982 and hence there was a net gain over the entire monitoring period.' was corrected to 'These post-1976 declines are lower than the $19.72 \%$ to $77.94 \%$ per decade increases observed in 1967-1976 (although these increases were not significant; Supplementary Table 1), and hence there was no net loss over the entire monitoring period.'

Seventh paragraph of main text, first sentence: 'Between-year changes in biomass confirm that there were several high-growth years in the mid- to late-1970s and two periods of consistent negative change in the 1990s (Fig. 2a).' was corrected to 'Between-year changes in biomass confirm that there were several high-growth years in the mid-1970s and two periods of consistent negative change in the 1990s (Fig. 2a).'

Seventh paragraph of main text, third sentence: 'Increases in biomass typically followed low biomass years, although declines following high biomass years were less evident (likelihood ratio test, $\boldsymbol{n}=\mathbf{1 2 3 8} ; \boldsymbol{R}^{2}=\mathbf{0 . 3 6}, \boldsymbol{\chi}^{\mathbf{2}}=\mathbf{2 2 2 . 0}$, d.f. $=1, P<0.001$; Fig. $2 \mathrm{~b}$ ).' was corrected to 'Increases in biomass typically followed low biomass years, although declines following high biomass years were less evident (likelihood ratio test, $\boldsymbol{n}=\mathbf{1 , 1 9 8 ;} \boldsymbol{R}^{2}=\mathbf{0 . 4 0}, \chi^{2}=\mathbf{2 4 7 . 2}$, d.f. $=1, P<0.001$; Fig. 2b).'

Seventh paragraph of main text, fourth sentence: 'Bayesian spectrum analysis found that about 3-5 yr elapsed between successive peaks in biomass change (highest peaks of the posterior distribution function were at 2.95, 3.40, 3.80 and 4.88 yr; mean of posterior distribution was $3.51 \mathrm{yr}, 95 \%$ confidence interval 2.07-11.26 yr; Extended Data Fig. 6), suggesting some pattern in the dynamics of measured biomass but not the cause.' was corrected to 'Bayesian spectrum analysis found that about 4-7 yr elapsed between successive peaks in biomass change (highest peaks of the posterior distribution function were at $2.35,4.81$ and $6.48 \mathrm{yr}$; mean of posterior distribution: $3.68 \mathrm{yr}$, $95 \%$ confidence interval: 2.03-19.29 yr; Extended Data Fig. 6), suggesting some pattern in the dynamics of measured biomass but not the cause.'

Eighth paragraph of main text, fourth sentence: 'Given that the relatively dry year of 1975 generated extreme population growth in $9 \%$ of lepidopteran species but the even drier year 1976 caused crashes in $>25 \%$ of species ${ }^{23}$, we suggest that the perturbation generated by these two years, followed by subsequent recovery and internal ecosystem feedbacks, underpins the rapid but short-term biomass growth seen in the late 1970s.' was corrected to 'Given that the relatively dry year of 1975 generated extreme population growth in $9 \%$ of lepidopteran species but the even drier year 1976 caused crashes in $>25 \%$ of species ${ }^{23}$, it is unsurprising that these years mark the peak of biomass, as estimated by segmented regression models.'

Eighth paragraph of main text, fifth sentence: 'Similarly, much of the post-1982 decline is accounted for by declines in the 1990s (Fig. $2 \mathrm{a} ;>10 \%$ of lepidopteran species 'crashed' in 1992/199323. was corrected to 'Similarly, much of the post-1976 decline is accounted for by declines in the 1990s (Fig. 2a; >10\% of lepidopteran species 'crashed' in 1992/199323.'

Eighth paragraph of main text, sixth sentence: 'However, the duration of the post-1982 reverse trajectories in woodland and grassland may indicate that other drivers are operating (for example, management, air quality ${ }^{24}$ or plant quality changes associated with $\mathrm{CO}_{2}$ levels and $\mathrm{N}$ inputs).' was corrected to 'However, the duration of the post-1976 reverse trajectories in woodland and grassland may indicate that other drivers are operating (for example, management, air quality ${ }^{24}$ or plant quality changes associated with $\mathrm{CO}_{2}$ levels and $\mathrm{N}$ inputs).' 


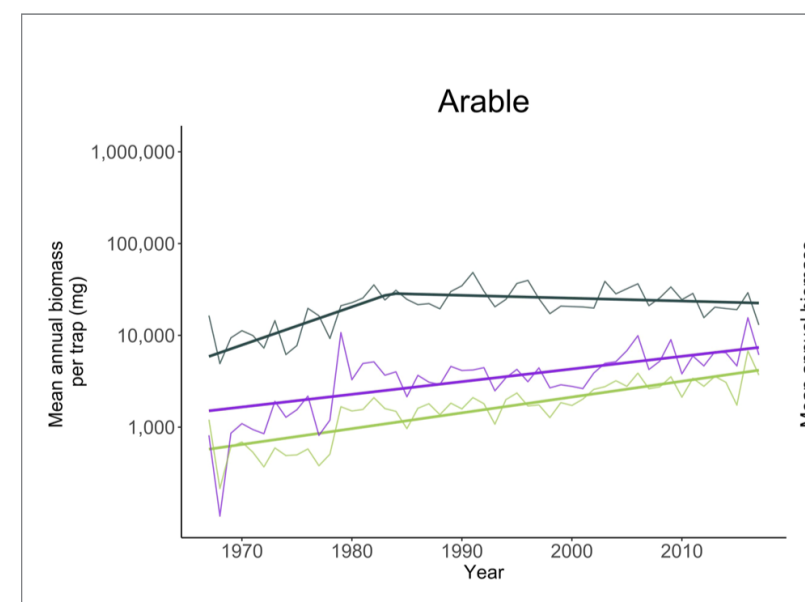

Urban
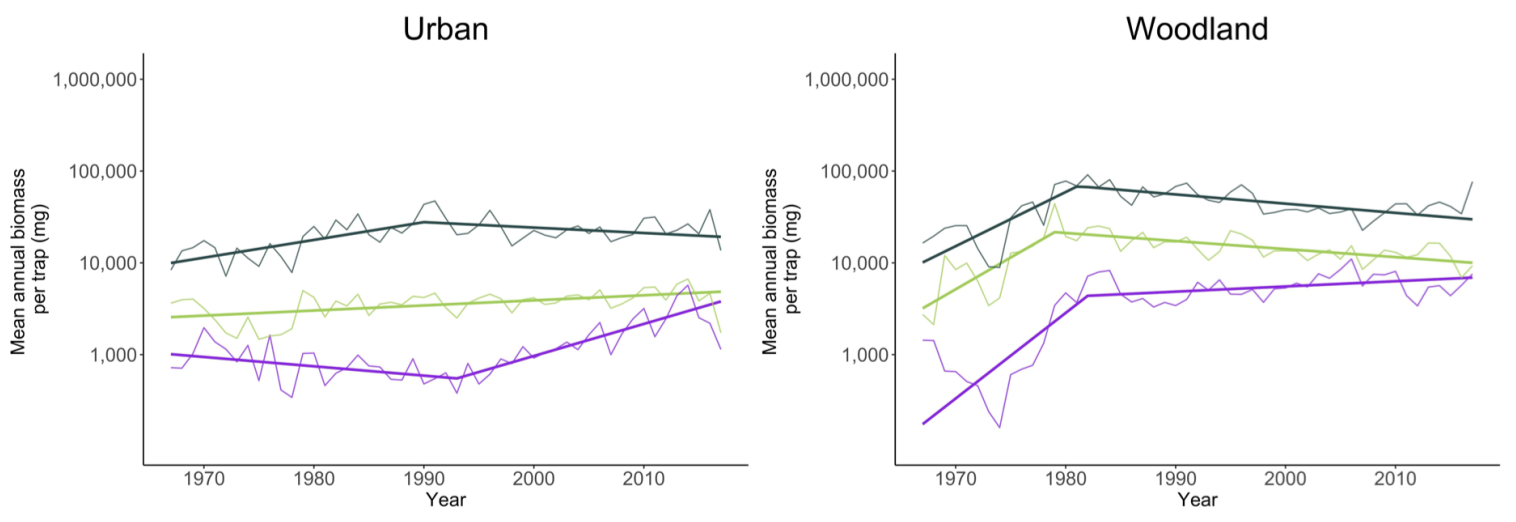

Corrected

Arable

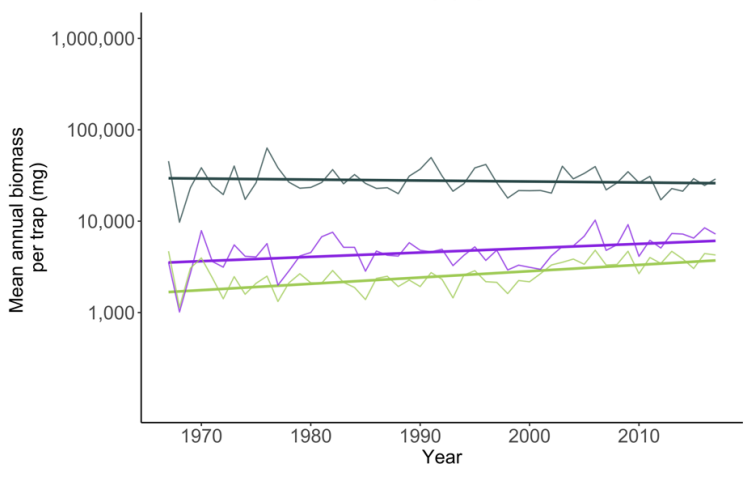

Urban

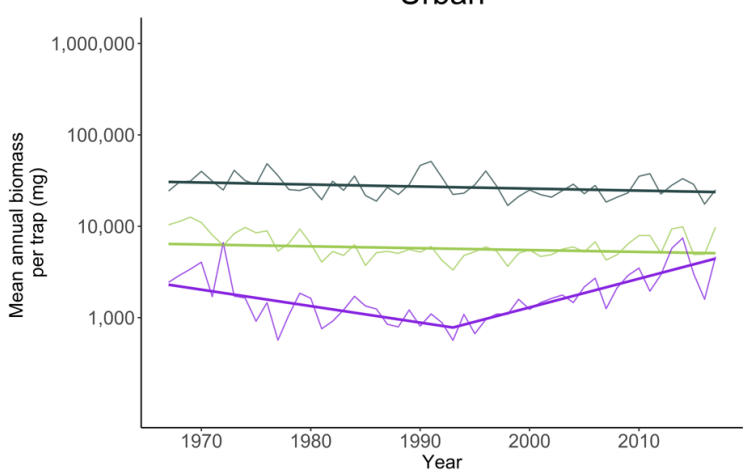

Extended Data Fig. 2 | Original and Corrected.

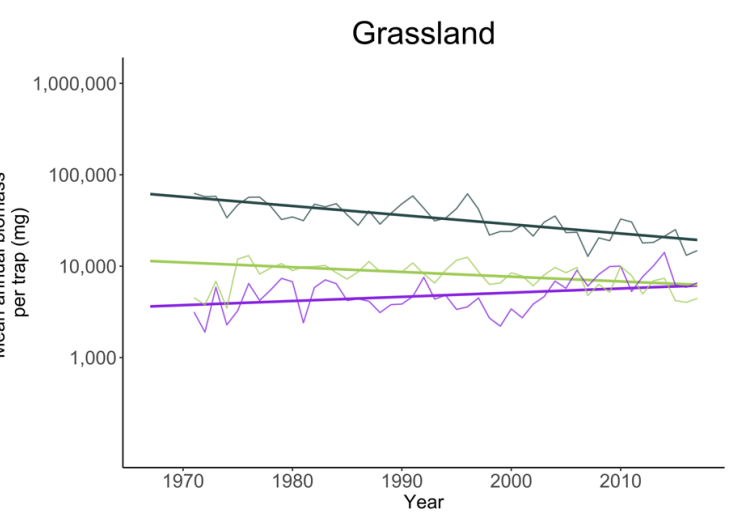

Woodland

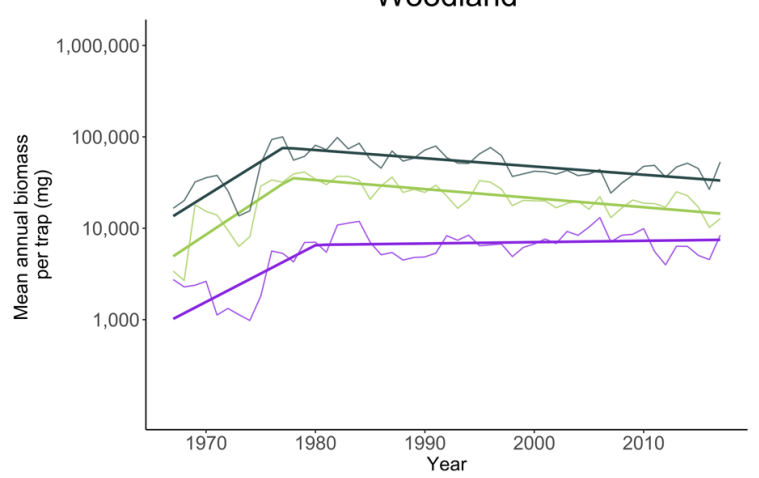

Grassland

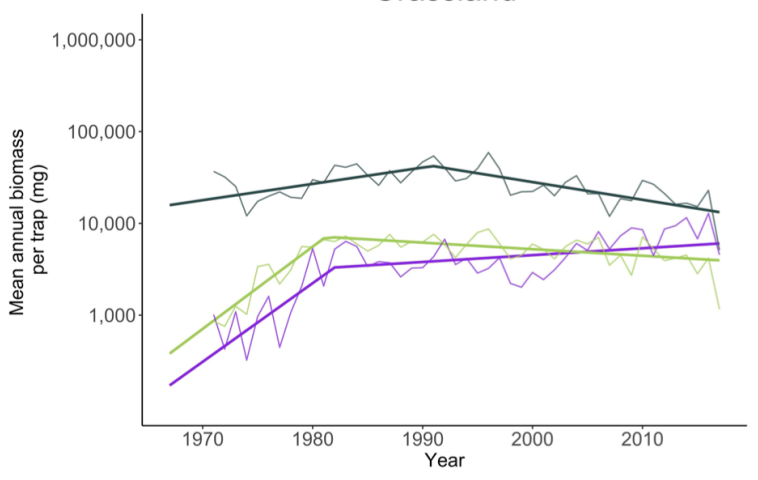

- Erebidae

- Geometrida

- Noctuidae
Family

- Erebidae

- Geometrida

- Noctuidae 


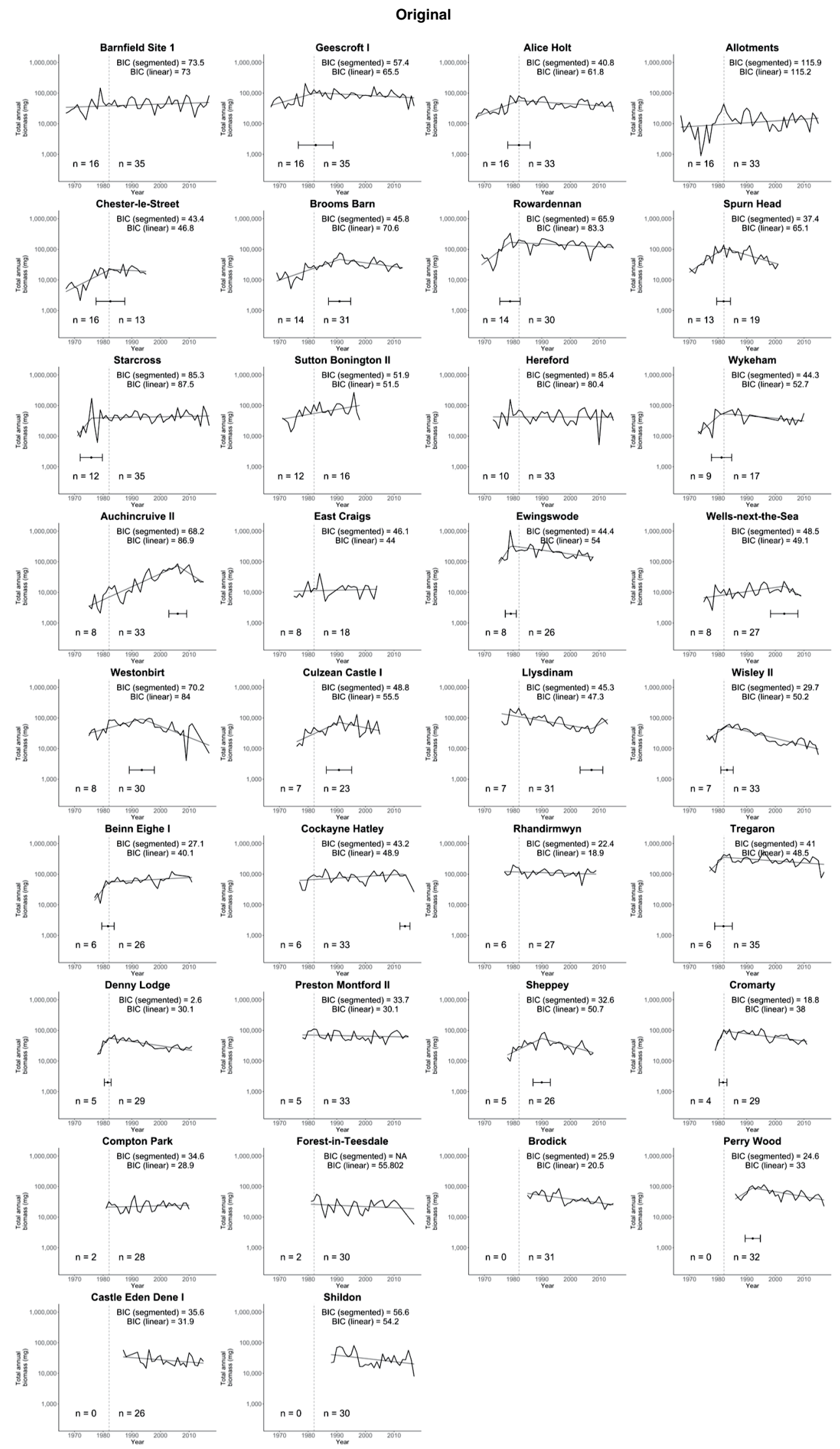

Extended Data Fig. 3 | Original and Corrected. 


\section{Corrected}
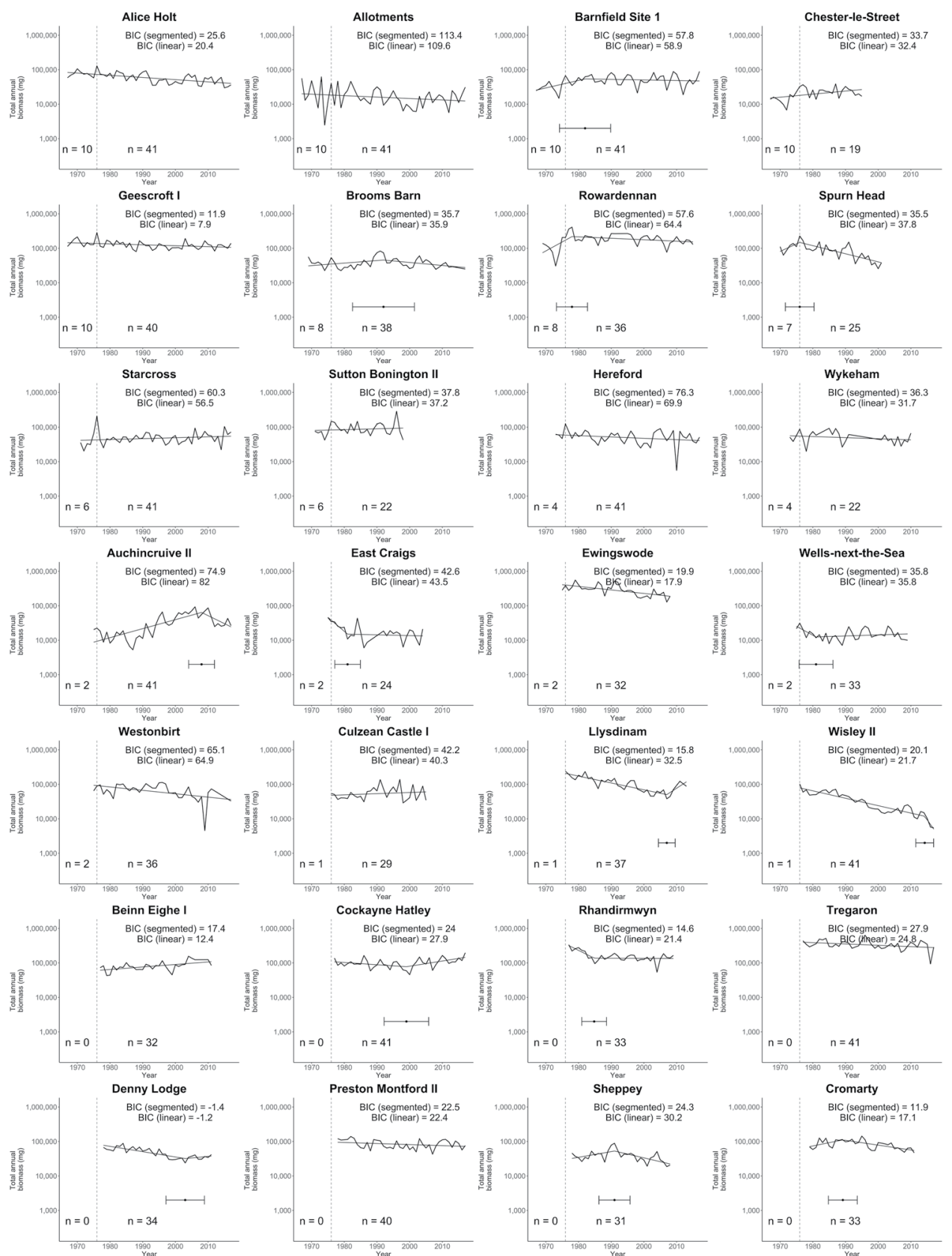

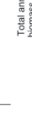
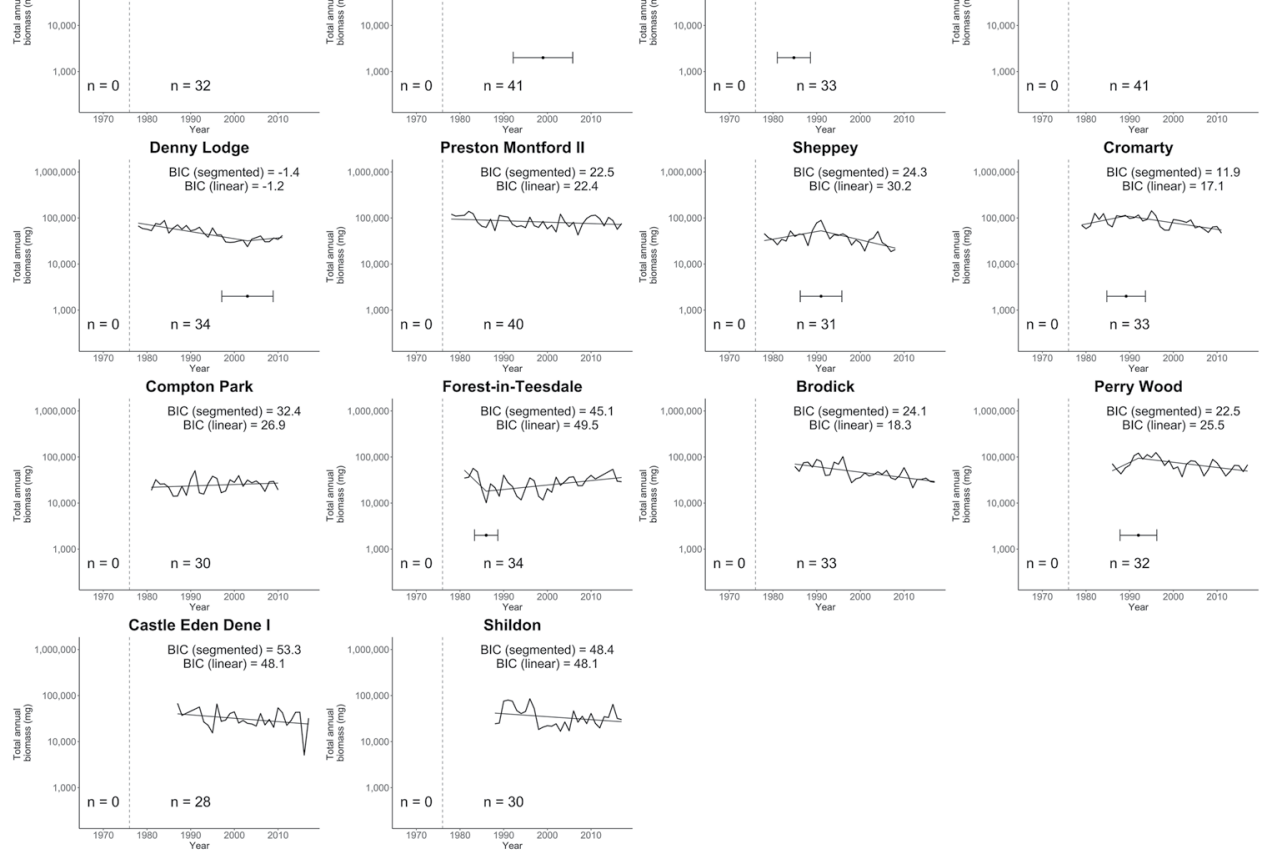


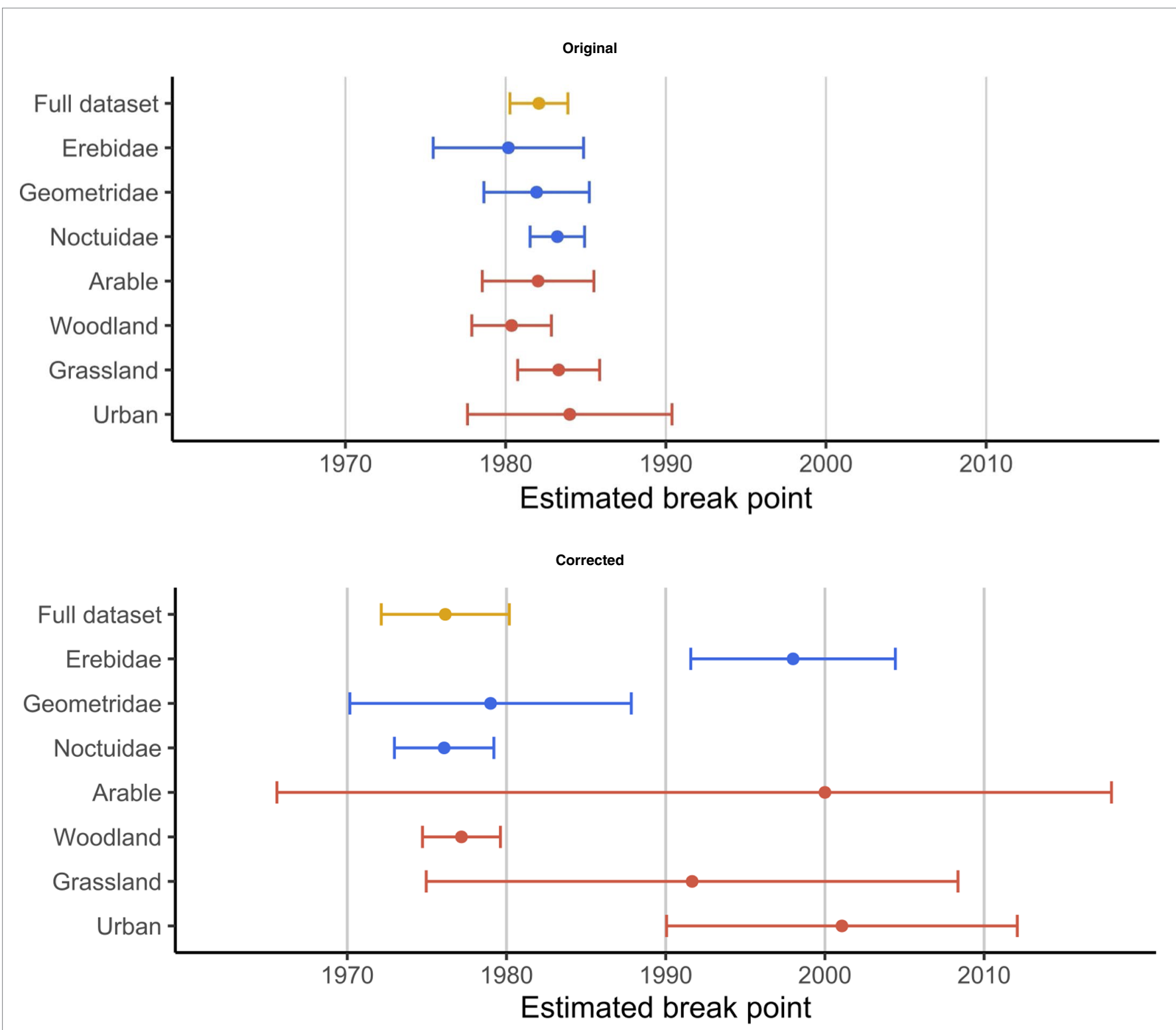

Extended Data Fig. 4 | Original and Corrected. 


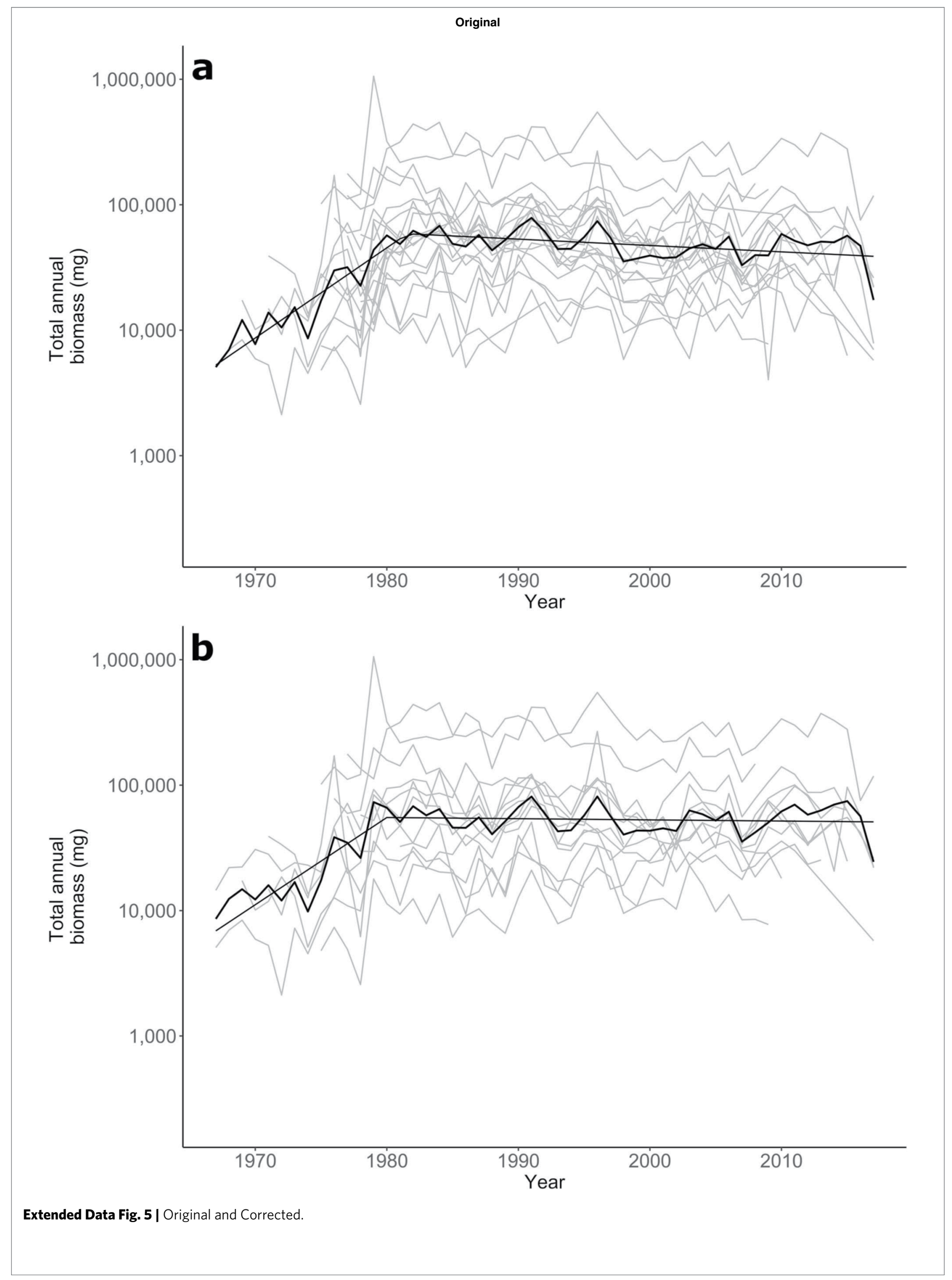




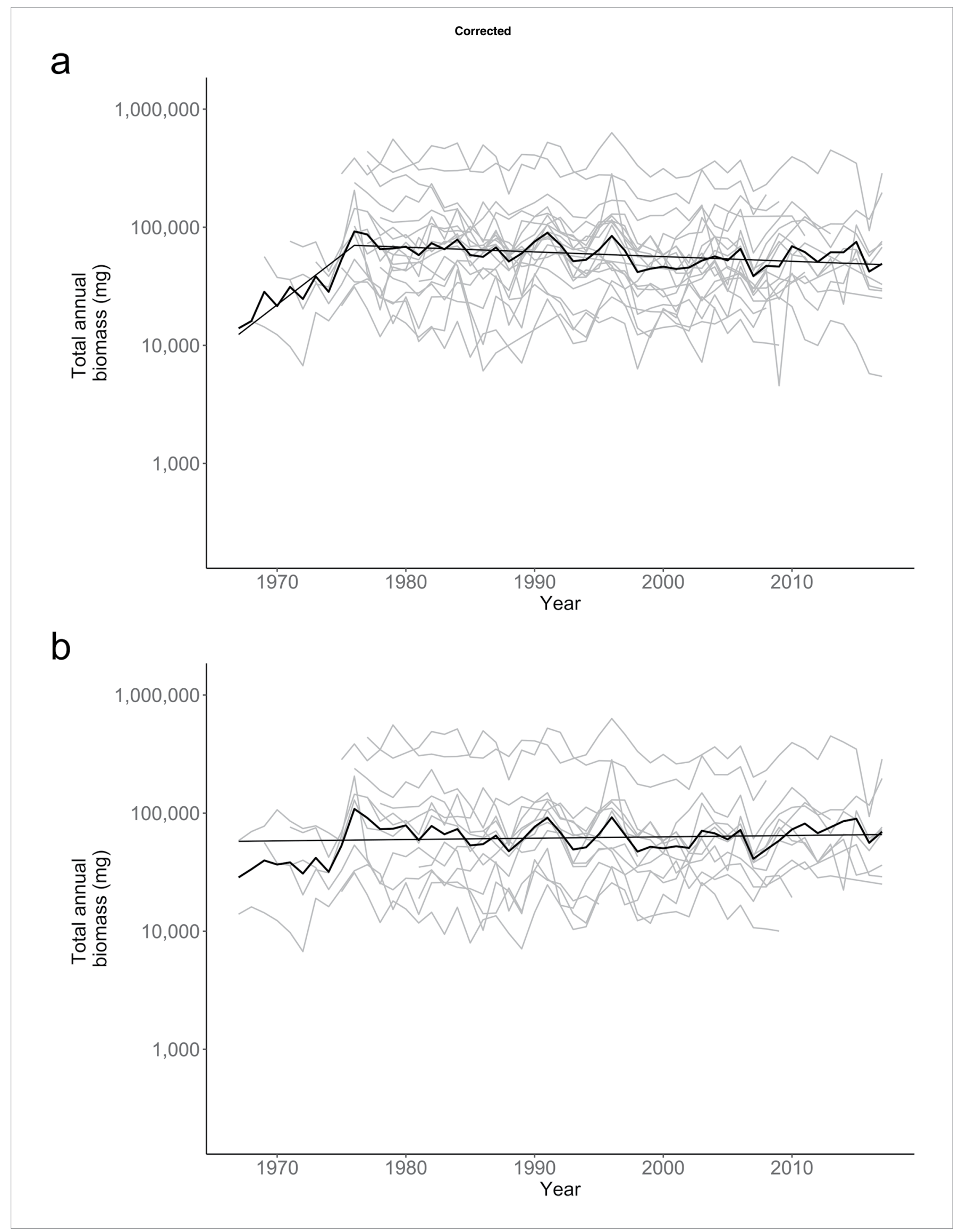



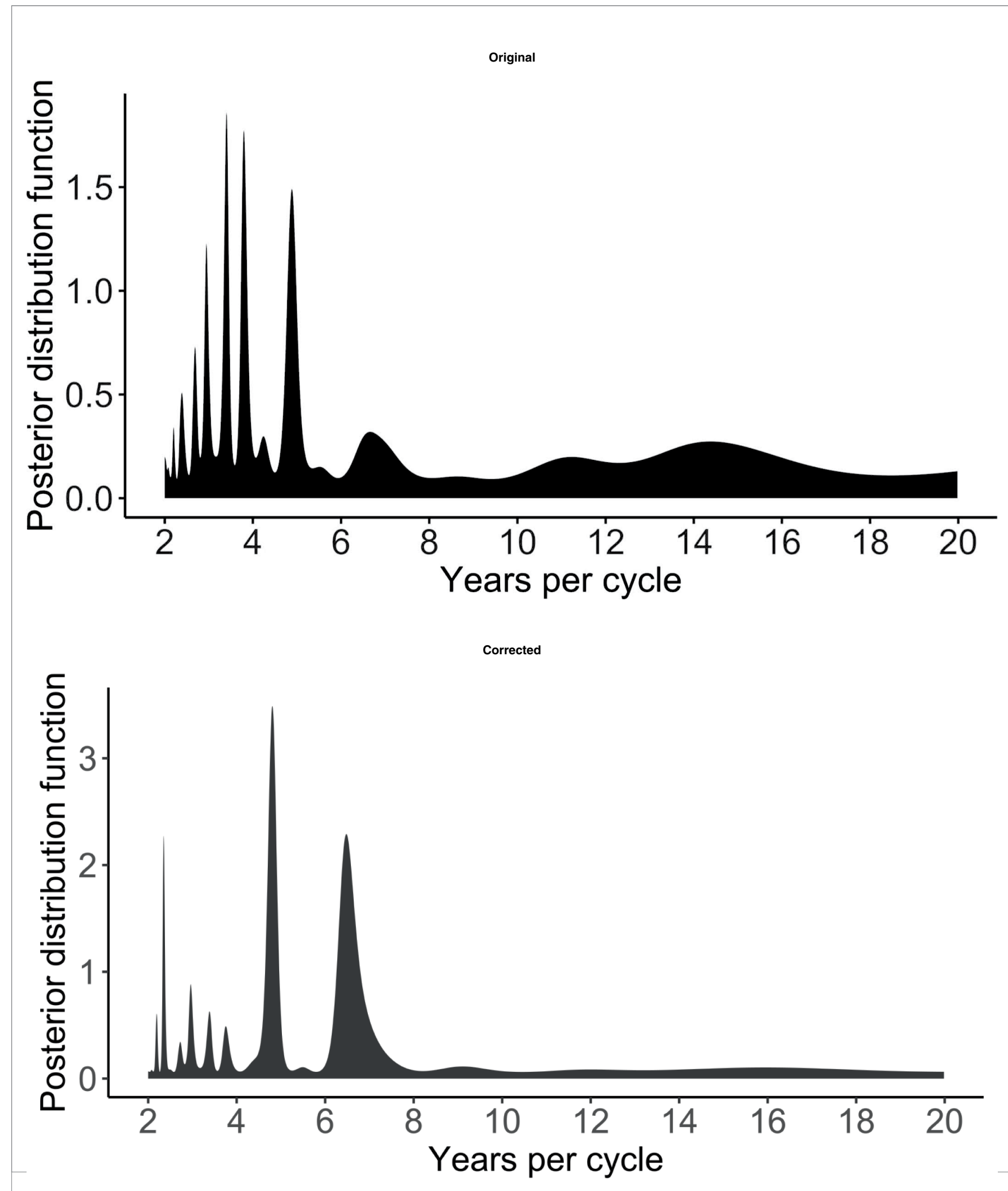

Extended Data Fig. 6 | Original and Corrected. 


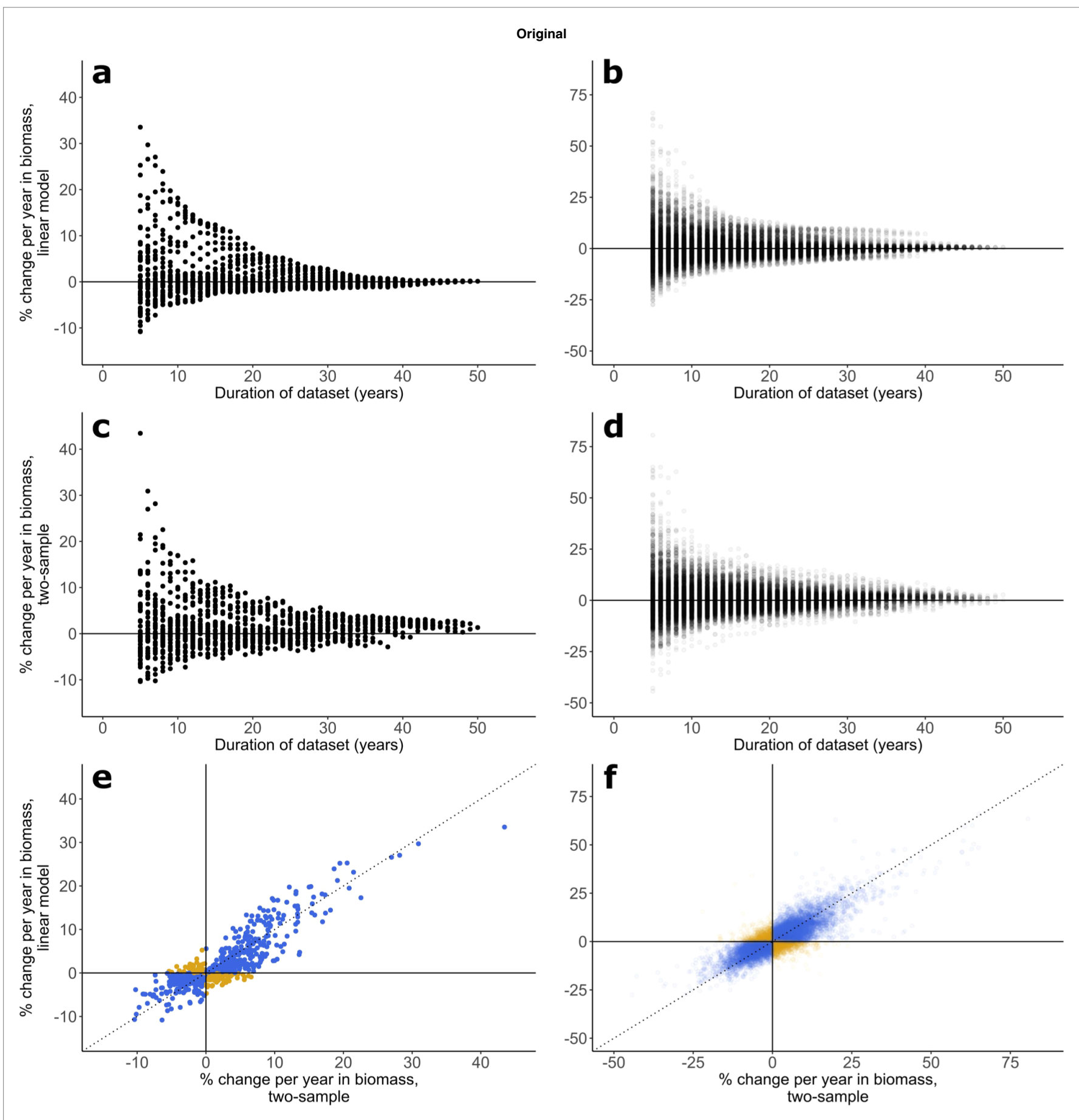

Extended Data Fig. 7 | Original and Corrected. 


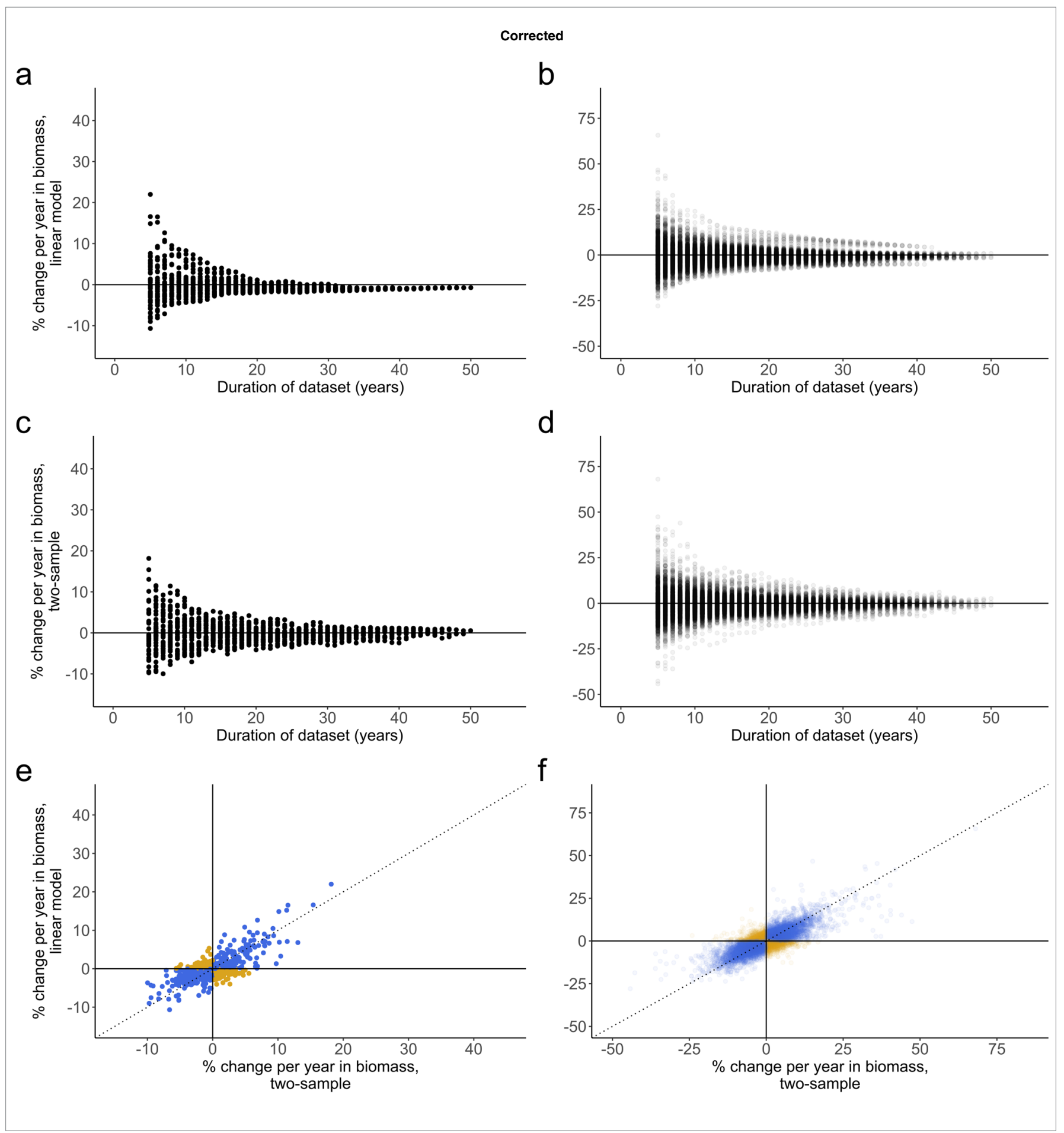




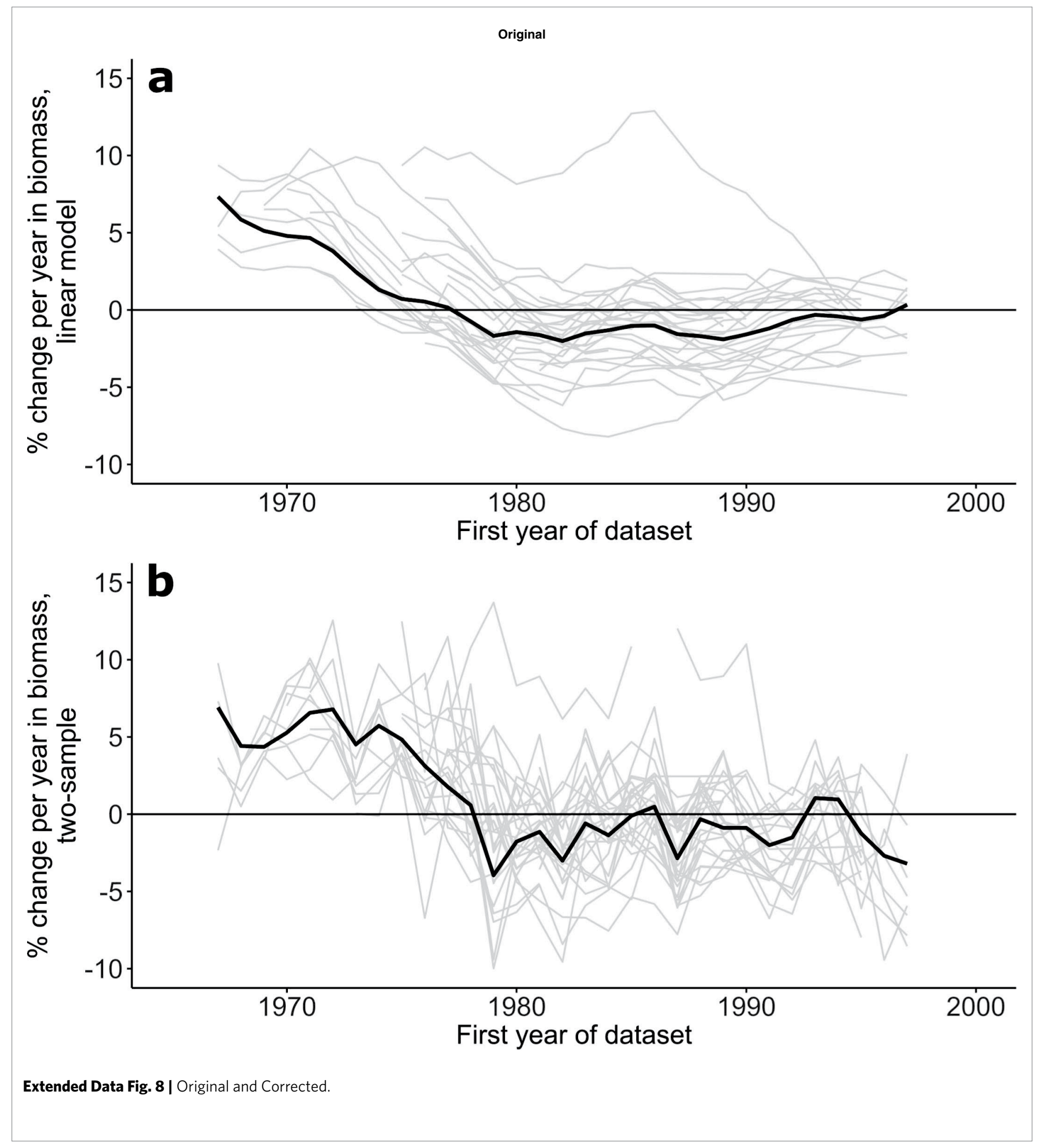




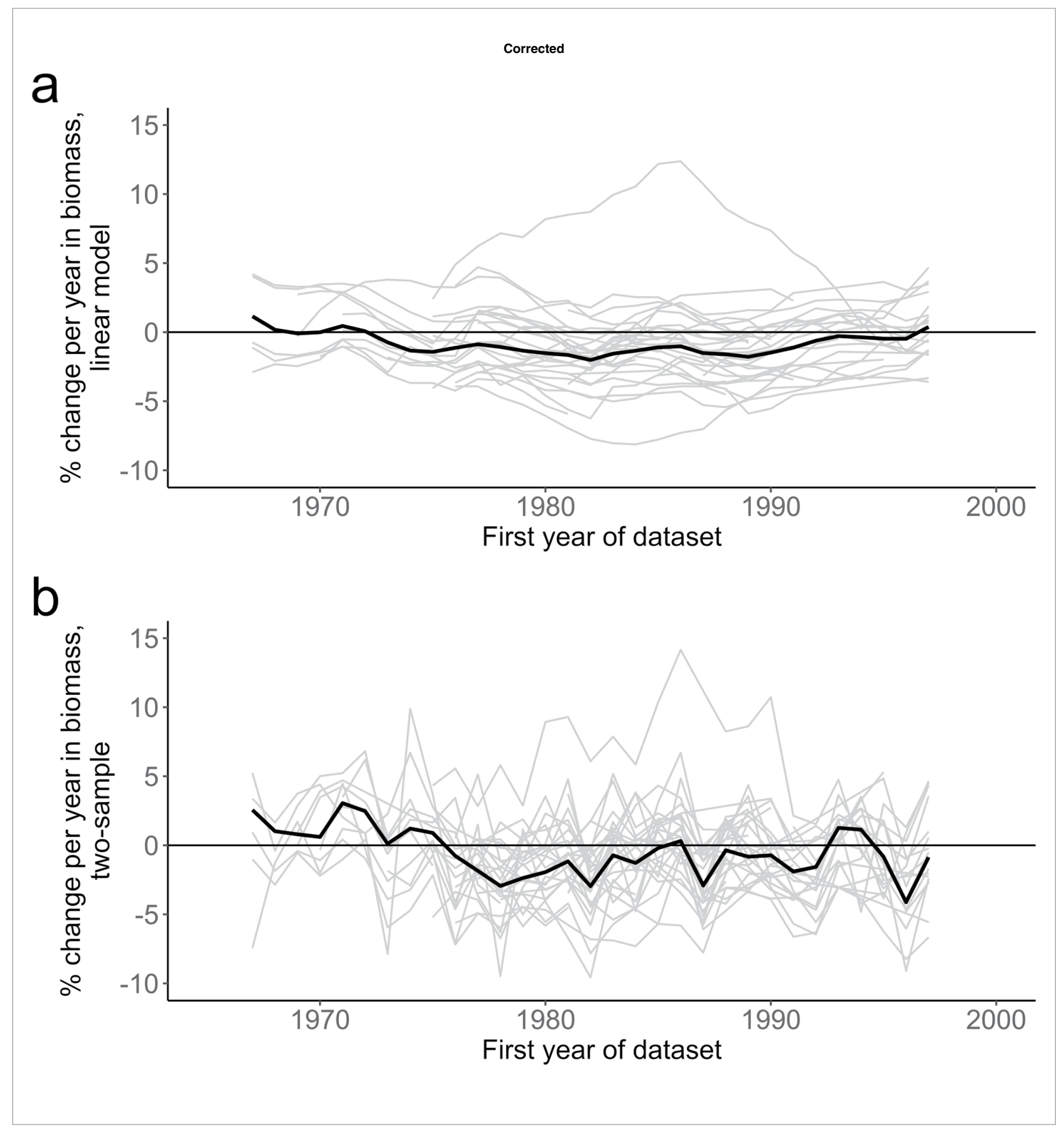




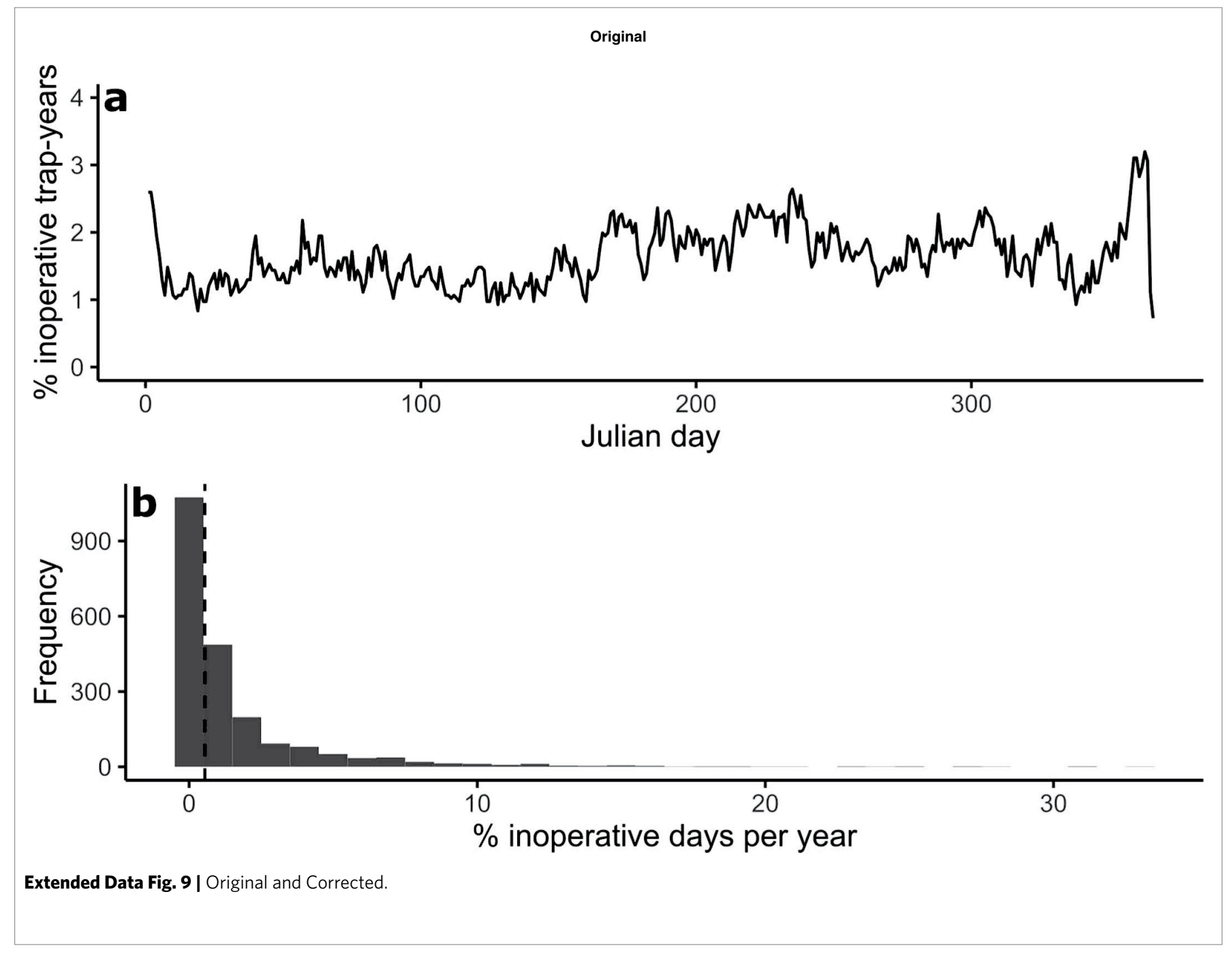




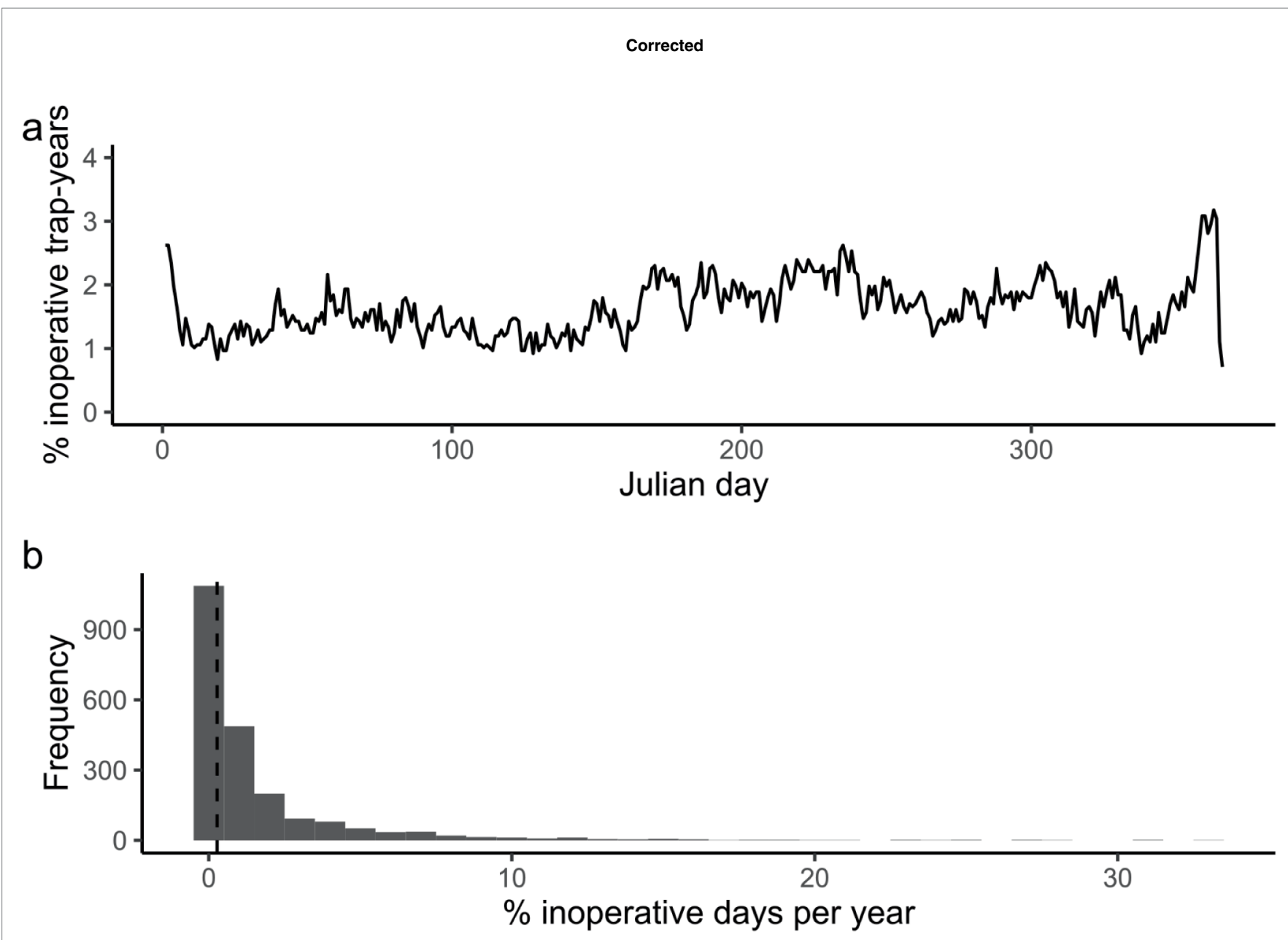

Ninth paragraph of main text, third sentence: 'Mean annual biomass (across traps) varied sixfold over the entire study period and about twofold (range 1.42-3.81) within each decade.' was corrected to 'Mean annual biomass (across traps) varied approximately twofold over the entire study period, and within each decade (range 1.44-2.69).'

Ninth paragraph of main text, fourth sentence: 'At individual traps, annual biomass varied up to 50-fold across the study period (range 3.29-49.26) and up to 28-fold (range 1.04-27.69) within each decade (Fig. 1a).' was corrected to 'At individual traps, annual biomass varied up to 25-fold across the study period (range 3.05-24.97), and within each decade (range 1.03-24.97; Fig. 1a)'.

Tenth paragraph of main text, first sentence: '...interval sampling (comparing first and last year of a sequence) incorrectly estimates the sign of regression-based 20-yr trends $\mathbf{2 4 \%}$ of the time (Extended Data Fig. 7e,f).' was corrected to '...interval sampling (comparing first and last year of a sequence) incorrectly estimates the sign of regression-based 20 -yr trends $\mathbf{2 9 . 1 \%}$ of the time $(\mathbf{2 2 . 1 \%}$ for individual traps; Extended Data Fig. 7e,f)'

Tenth paragraph of main text, second sentence: 'Equally, the start (baseline) date is critical; the slopes of 20-yr trends depended on when a time series started, relative to the $\mathbf{1 9 8 2}$ peak...' was corrected to 'Equally, the start (baseline) date is critical; the slopes of 20-yr trends depended on when a time series started, relative to the 1976 peak...'

Eleventh paragraph of main text, first sentence: 'In conclusion, we showed that post-1982 decline in the biomass of British moths was preceded by a larger increase.' was corrected to 'In conclusion, we showed that post-1976 decline in the biomass of British moths was preceded by a comparable increase.'

Eleventh paragraph of main text, third sentence: 'The decline in the post-1982 period...' was corrected to 'The decline in the post-1976 period...'

Methods, second paragraph of 'Linear and segmented regressions' subsection, second sentence: 'In addition to the full dataset, we analysed subsets of data: (1) separating data for the three most abundant families of moths (Erebidae, Geometridae and Noctuidae), which collectively comprised $\mathbf{9 3 . 3 \%}$ of total biomass in our dataset... was corrected to 'In addition to the full dataset, we analysed subsets of data: (1) separating data for the three most abundant families of moths (Erebidae, Geometridae and Noctuidae), which collectively comprised $\mathbf{9 0 . 8 \%}$ of total biomass in our dataset...' 
Methods, second paragraph of 'Linear and segmented regressions' subsection, sixth sentence: 'In eight out of ten comparisons, the BIC and the likelihood ratio test were in agreement that the segmented model was the better fit; in the remaining two, the likelihood ratio test favoured the segmented model but the BIC was marginally lower for the linear model (Supplementary Table 1).' was corrected to 'In eight out of ten comparisons, the BIC and the likelihood ratio test were in agreement which model was the better fit; in the remaining two, the likelihood ratio test favoured the segmented model but the BIC was marginally lower for the linear model, suggesting models fitted similarly well (Supplementary Table 1).

Methods, third paragraph of 'Linear and segmented regressions' subsection, first sentence: 'Finally, to confirm the significance of biomass trends (both overall and in each land-use class) before and after the universal inflection point (estimated at 1982; Supplementary Table 1), we split the dataset into early (1967-1982) and late (1983-2017) periods.' was corrected to 'Finally, to test the significance of biomass trends (both overall and in each land-use class) before and after the universal inflection point (estimated at 1976; Supplementary Table 1), we split the dataset into early (1967-1976) and late (1977-2017) periods.'

Figs. 1 and 2: the data were corrected for both figures and the following sentence was added to the end of the Fig. 1 caption: 'In $\mathbf{d}$ only, the significance of trends is indicated by solid lines (non-significant trends are represented by dashed lines).'

Extended Data figures: the data were corrected for Extended Data Figs. 2-9 and the following Extended Data figure captions were amended:

Extended Data Fig. 1 caption, first sentence: '...the duration of trapping at each location is indicated by shape (downwards-pointing triangle: eight traps commencing before 1970, with > 12 years of data before the general inflection point; upwards-pointing triangle: 26 traps commencing after 1970, with 12 or fewer years of data before the general inflection point).' was corrected to '...the duration of trapping at each location is indicated by shape (downwards-pointing triangle: eight traps commencing before 1970 , with $>\mathbf{6}$ years of data before the general inflection point; upwards-pointing triangle: 26 traps commencing after 1970, with 6 or fewer years of data before the general inflection point).'

Extended Data Fig. 3 caption, third sentence: 'The inflection point from the overall model (1982.08) is shown as a dotted line...' was corrected to 'The inflection point from the overall model (1976) is shown as a dotted line...'

Extended Data Fig. 3 caption: the final sentence 'For Forest-in-Teesdale, segmented model-fitting failed to converge under all conditions, so the linear model is shown.' was removed.

Extended Data Fig. 4 caption: the final sentence 'The central estimate for the full dataset lies within the 95\% CI of the estimate for all subsets of the data.' was removed.

Extended Data Fig. 5 caption, fifth sentence: 'Black line depicts the trend fitted by a segmented regression.' was corrected to 'Black line depicts the trend fitted by the best-fitting model from a segmented regression and a linear regression.'

Extended Data Fig. 8 caption, fourth sentence: 'Twenty-year time sequences that originate before the late 1970s (that is, well before the 1982 peak) consistently suggest increases in biomass over time, whereas sequences originating later than this typically indicate declines in biomass.' was corrected to 'Twenty-year time sequences that originate before the late 1970s (that is, before the 1976 peak) suggest increases in biomass over time, whereas sequences originating later than this typically indicate declines in biomass.'

The URL in the Code availability statement was amended from 'https://doi.org/10.5281/zenodo.3356841' to 'https://doi.org/10.5281/ zenodo.4442298'.

An additional sentence was added to the end of the Acknowledgements section: 'We thank C. Harrower, M. Pocock, D. Blumgart and C. Shortall for their help in bringing the data error in the originally published version of this Brief Communication to our attention and investigating its cause.'

In the Supplementary Information, Supplementary Tables 1-6 and Supplementary Dataset 1 were replaced with all values corrected.

All errors have now been corrected.

Published online: 14 May 2021

https://doi.org/10.1038/s41559-021-01449-5

(C) The Author(s), under exclusive licence to Springer Nature Limited 2021 